\title{
Research on College English Video Learning System Based on Streaming Media Technology
}

\author{
Liu Dan \\ Teaching and Research Institute of Foreign Languages, Bohai University, Jinzhou, 121013, China \\ myld81@163.com
}

Keywords: streaming media technology; College English; video learning system; system architecture; system function; system implementation

\begin{abstract}
Streaming media is a, downloading while playing, immediately streaming technology that used in the continuous time base media streaming transmission technology to transmit data stream. This article is based on streaming media technology research, provide a new research scheme for the video of college English learning system. First, research media encoder, media file storage, and media players such as streaming media server system architecture; Next, research streaming media technology, illustrates the streaming process; Then, design a function structure consists of side teachers side and students side and management side, etc; Finally, study the key technology of the live broadcast system implementation. In this paper, the research results laid a foundation to the development of the software.
\end{abstract}

\section{Introduction}

Streaming media is refers to the streaming technology continuous real-time broadcast media format on the Internet, such as audio, video, or multimedia files. Streaming media technology is the continuous image and sound information, after dealing with the compression on the web server, from the video server to the user's computer order or each package in real-time transmission, allows users to download and watch or listen to, but don't wait to download the zip file to your computer to watch network transmission technology. The technology to create a buffer on the user's computer, playing a piece of data as a buffer in advance before actual connections on the network speed is less than the speed of broadcast by, broadcast application can access data in a buffer zone, so that you can avoid interruption, will be broadcast quality guarantee.

College English teaching is an integral part of higher education, college English course is a required basic course of college students. College English is a foreign language teaching theory as the guide to English language knowledge and application skills, cross-cultural communication and learning strategy as the main content, and collect a variety of teaching mode and teaching means for the integration of teaching system. Each institution of higher learning should make full use of modern information technology, based on the computer and the classroom English teaching mode, the improvement is given priority to with teachers teach single teaching mode. New teaching model should be to modern information technology, especially network technology as the support, can make the teaching and learning of English in a certain extent, not limited by time and place, the direction of individualized and autonomous learning. New English teaching mode should reflect the principle of combining practical, informative and interesting teaching, is helpful to arouse the enthusiasm of teachers and students two aspects, especially want to reflect the subject status of students in the teaching process and teachers in a leading role in the process of teaching. In making full use of modern information technology at the same time, reasonable to inherit the outstanding part of the traditional teaching mode, play the advantages of traditional classroom teaching [1, 2].

Network video teaching system combines the advantages of multimedia technology and network technology, accepted by educators can according to their own ability, professional level and ability to understand and make full use of network resources for learning, so as to make the education breaks through the traditional education mode of space and time limit, has realized the student's 
individualized education. In this paper, based on streaming media technology, combined with the characteristic of English study, to provide technical support for the system development.

\section{System Architecture on Streaming Media}

Streaming media system can be divided into four parts: the media encoder, media file storage, media server, and media players, four parts collaborate streaming media service system. The parts according to the specific format data exchange file. System architecture and the relationship among each part is shown in Fig. 1.

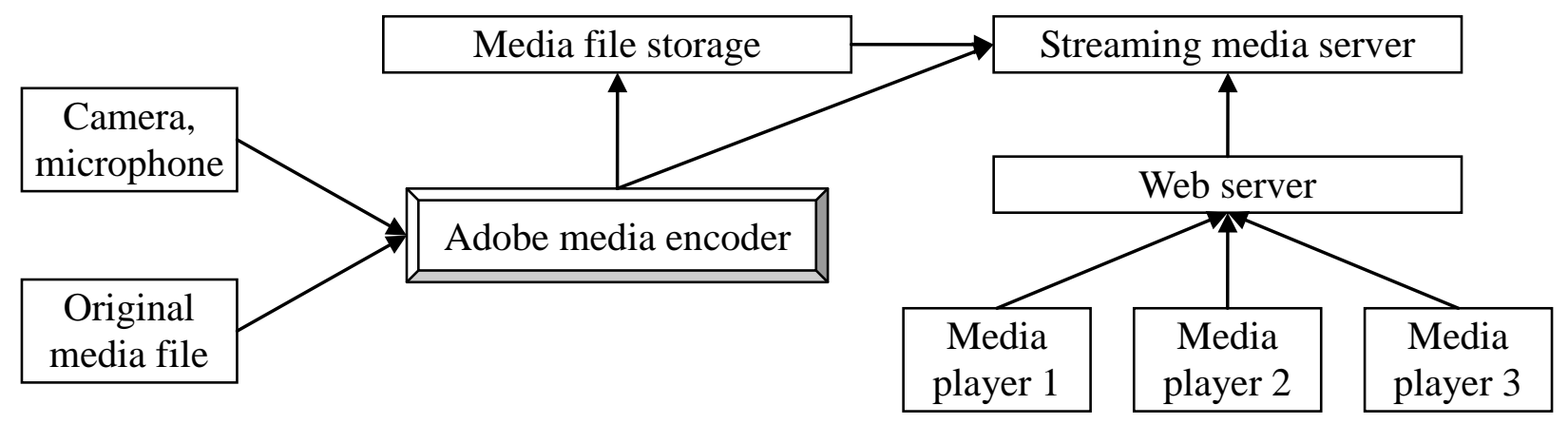

Fig. 1. Streaming media system architecture

(1) Media encoder: the original media file or camera acquisition in real-time media data, made suitable for network transmission of the file format (stream format), and then will be streaming media files stored on the media server, or directly to the streaming media server.

(2) Media file storage: storage stream media file formats, generally USES SCSI hard disk or disk array.

(3) Media server [3] : user request response from a Web server, through the network transmission protocol will stream format of the file to the user's desktop.

(4) Media player: receive network media data and broadcast on the local.

\section{Technological Principles on Streaming Media}

The realization of the streaming transmission need to cache. As a source of real time audio and video or audio and video files stored in the transmission is broken into packets, the network is dynamic, each package selection routing may not be the same, time delay are different, so the client even starts packets may arrive after. Therefore, you need to use the caching system to eliminate the influence of time delay and jitter, to ensure correct packet sequence, thus enables the media data to continuous output.

The realization of the streaming need appropriate transport protocol. Because TCP requires more overhead, it is not suitable for real-time data transmission. In the realization of the streaming scheme, generally using HTTP/TCP to transmit control information, and using real-time transport protocol/user datagram protocol (RTP/UDP) to transmit data in real time. Streaming the basic principle is shown in Fig. 2.

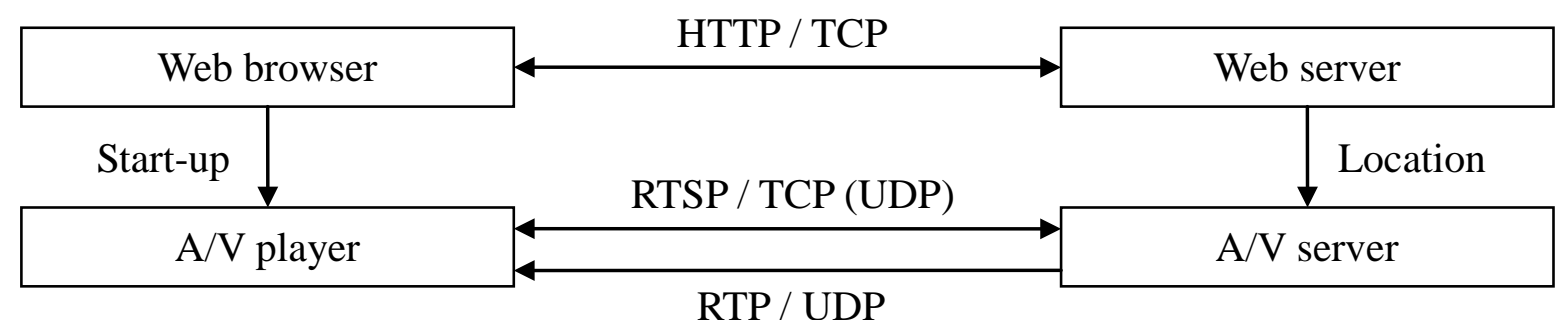

Fig. 2. Basic principle of stream transmission

Streaming process specification is as follows:

(1) After the user to select a leading media services, between the Web browser and the Web 
server using HTTP/TCP control information exchange, in order to transmit the need of real-time data retrieved from the original information;

(2) Web browser to start the AV client, using HTTP from a Web server to retrieve relevant parameters on the AV client application initialization, these parameters may include directory information, audio and video data encoding type or the server address associated with audio and video retrieval;

(3) Audio and video and audio video server running real-time streaming protocol client, in exchange for audio and video transmission control information needed, real-time streaming protocol provides play, fast forward, fast down, pause and recording of the command method;

(4) Audio and video server using RTP/UDP (real-time transport protocol/user datagram protocol) to audio and video data transmission to AV client, arrived at the client once the audio and video data, audio and video client programs can be output.

To be sure, in the transmission of streaming, and RTSP using RTP/UDP/TCP two different communication protocols with audio and video server connection, purpose is output to the server to run an audio video client destination address of the client. In addition, implement streaming typically require a dedicated server and player.

\section{Design on System Function}

Design on System Function is conducted after the feasibility study and demand analysis, is the core of the profile design work. Functional design should follow the principle of information hiding and module independence, reduce the coupling, improve the degree of cohesion, the design results is to use the structure module hierarchy and call relationship. Users of English learning video systems including students, teachers and administrators, students is the core of users, via mobile devices to complete English learning; Teachers and administrators is auxiliary the user for the system to provide the resources and ensure the system run [4-6]. System functions according to the management side, the side of the teachers and students and design, module hierarchy is shown in Fig. 3.

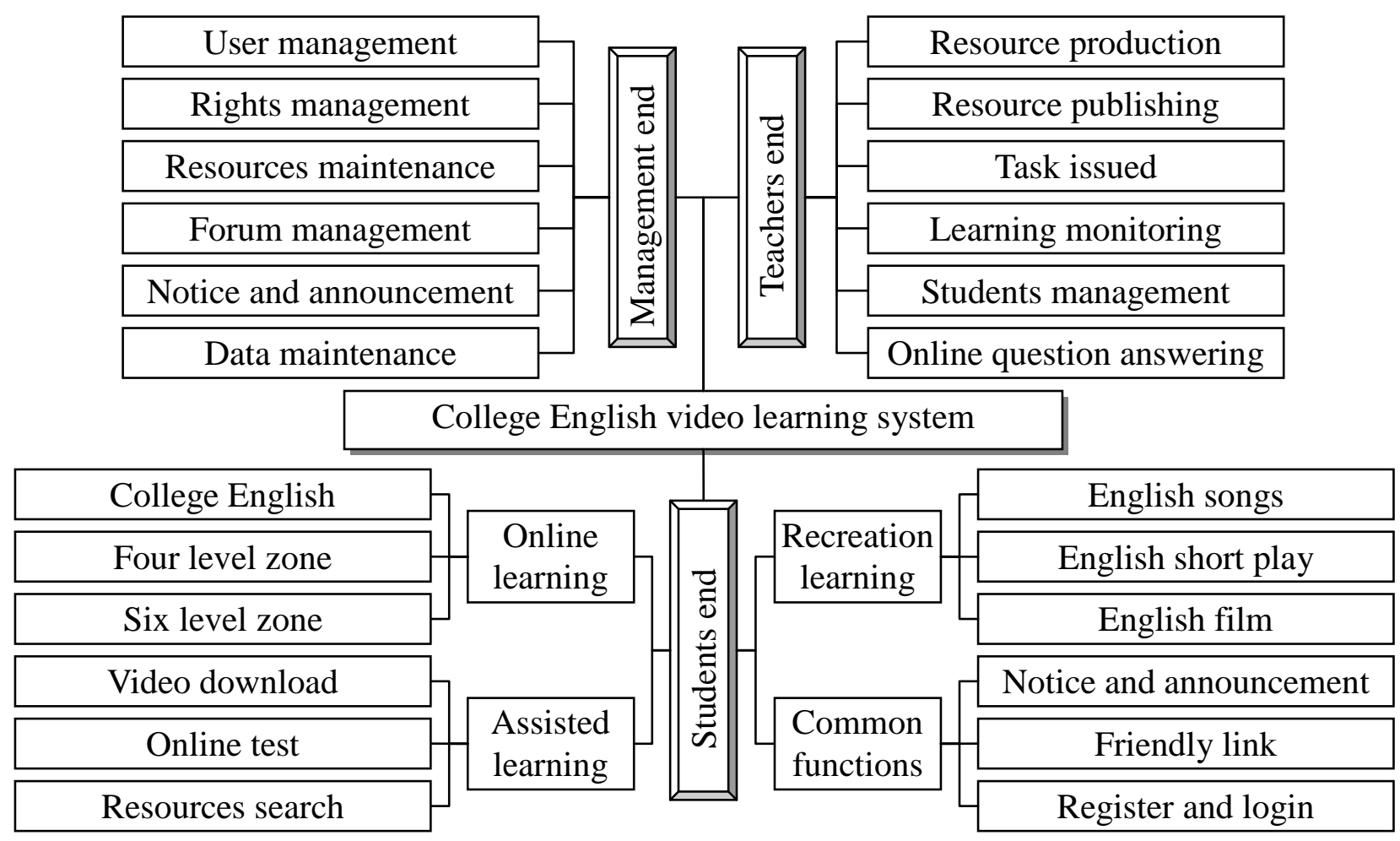

Fig. 3. Hierarchical structure on functional module for college English video learning system

Shown in Fig. 3, the function modules of the brief description is as follows: 
(1) Management side. Provide for the use of the system administrator to maintain normal operation of the system. Mainly to complete the user registration, roles and authorization management; Classifying resources and maintenance; Review and delete of BBS information; Notice notice; The data backup and restore, etc.

(2) Teacher side. Provide for the use of teachers, is used to provide video learning resources and management of students' learning. The main complete released video production and resources; From learning tasks and to monitor the learning situation; The student information management and answer students questions online.

(3) Students side. Provide for the use of students, the main function is divided into four categories: one is that online learning, is the core of the system function, provide video resources including college English Test-4,6, etc.; Two is, the entertainment study, in the entertainment at the same time to finish learning English, expand students' knowledge and cross-cultural ability, including songs, plays and movies and other video; Three is, assisted learning and improve the video download, online test and resource search function; Four is the general function of the system is normal function, other users can use, including notice notices and links.

\section{Implementation on Living Broadcast System}

Implementation on Living Broadcast System refers to the original data for H264 encoding and AAC encoding, audio and video data encapsulation for MPEG - TS packet, HLS piecewise strategy and m3u8 index file, the HTTP transport protocol, such as the five key technologies, research in this section, two related technologies of HLS.

(1) HLS logical process. Live video, for example, realization of HLS LiveEncoder encoder logical process as shown in Fig. 4. Basic process is open audio and video encoding thread respectively, through the DirectShow technology to realize audio and video collection, and then call libx264 and libfaac respectively for video and audio encoding. After two coding threads real-time coding audio and video data, according to the custom of the subdivision strategy, stored in a MPEG - TS file format section, when finish a fragment file storage, update m3u8 index file.

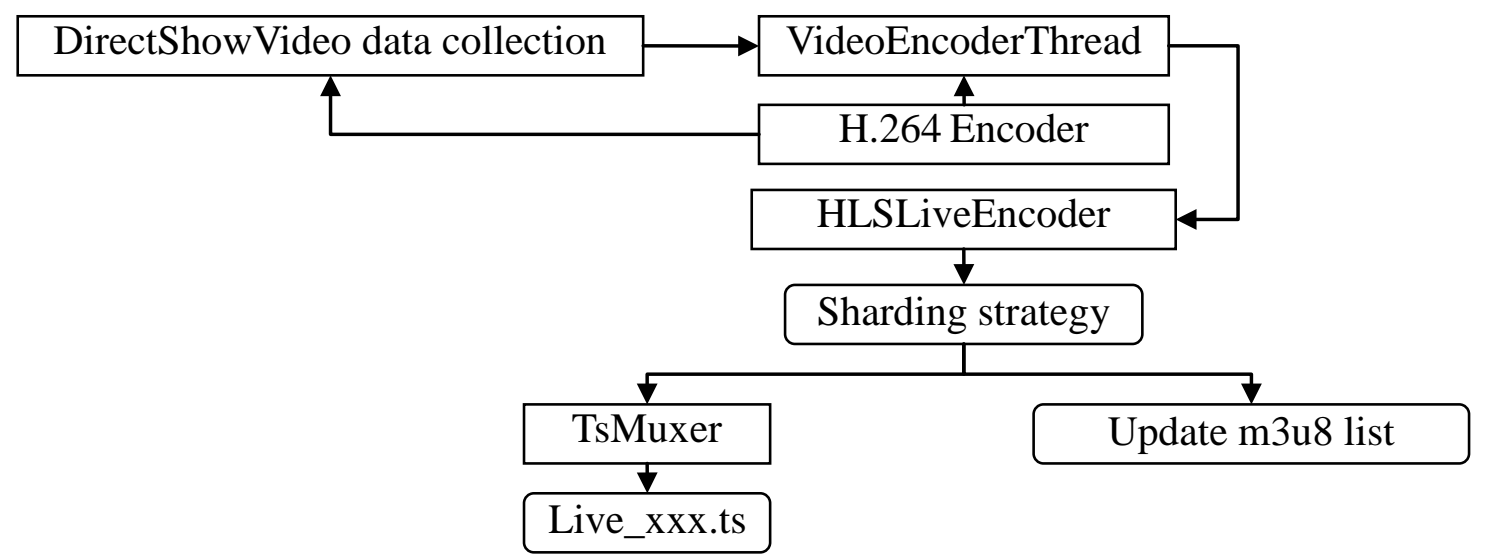

Fig. 4. HLS logical flow

(2) HLS class implements. HLS implementation class is shown in Fig. 5 [7]. CHLSServer, carries on the Socket to listen to, manage client HLS requests; CHLSClient, realizes the HTTP request and response, according to the client request of HLS content, to CHLSSource processing; CHLSSourceManager, all source of HLS manager, HLS source can be a local file, also can be real-time data from the network or device; CHLSSource, implement specific HLS requests, including M3U8 file access and TS fragments.

\section{Conclusion}

The traditional classroom teaching due to the time dependence and spatial dependence, makes the imparting of knowledge update slow, without representation and playback function; Restricted by 
space and time can't meet the requirements of people learning anytime and anywhere; Taught knowledge comprehensive and three-dimensional cross is lacking. And video teaching breaks through the traditional classroom teaching in time and space limitations, increased the learning opportunities, reducing the costs of learning, students can according to their own needs to learn anytime and anywhere, so as to improve the teaching quality. As supplement and extension of classroom teaching and video teaching will become an inevitable direction of the development of the education of colleges and universities in the future. Will take the student as the main body of teaching system in the future, make full use of voice, video capture the real classroom scene, make teachers and students in the state of separation, using the synchronous video technology and interactive voice technology, still can face to face communication and communication, so as to establish a harmonious and harmonious network teaching environment, promote each other's feelings, enhance the confidence of the students to participate in online learning, arouse the enthusiasm of learning. According to this article research results the development of the successful application of college English learning video system, to improve the level of college English teaching, promote the remote education is of great significance in such aspects.

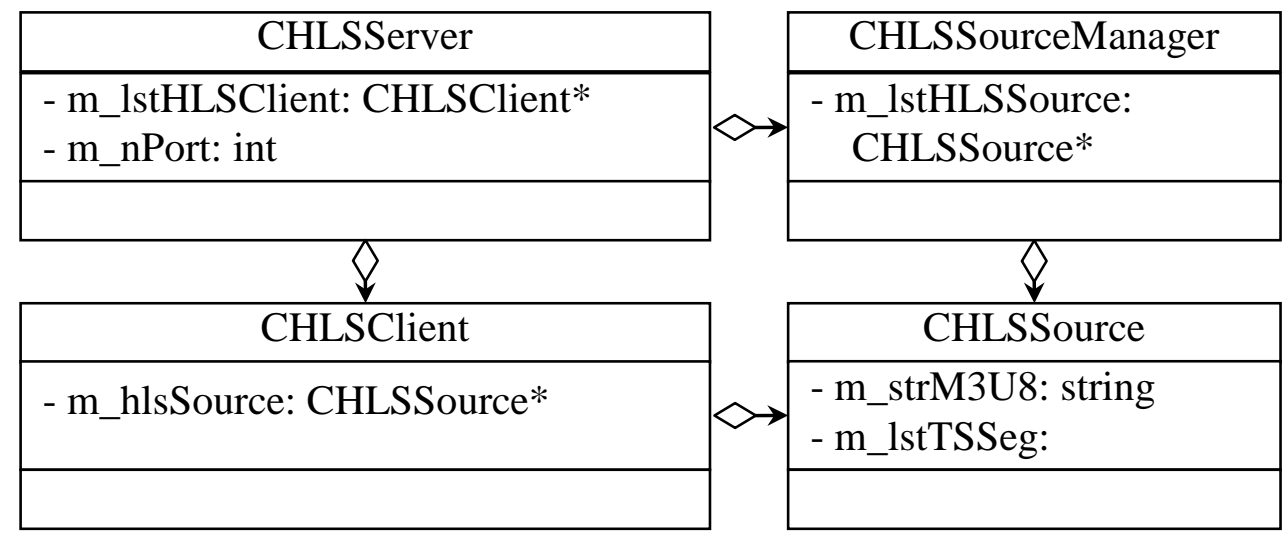

Fig. 5. HLS implementation class diagram

\section{References}

[1] C. Z. Mai, "Research and application of streaming media technology based on media windows in Teaching," Management and technology of small and medium sized enterprises, vol. 6, no. 2, pp. 250-251, 2013.

[2] Baidu Encyclopedia, "College English Curriculum Requirements," http://baike.baidu.com/link?url=2eyGJpDNAyAL0v5OlLkZNeI9Lx7jqjE1ZEs0A5iM_u7Z-KE eMoX9epSMGrPFHYe7vg5g5VJHXoZoBqqW9iLRiK, 2016-2-10.

[3] Jeffrey D. Weekley, Cees de Laat, "CineGrid, high quality media streaming and processing on advanced photonic networks," Future Generation Computer Systems, vol. 54, no. 1, pp. 292-295, 2016.

[4] Y. Cao, L. Chen, J. L. Wang, et al, "Design of Mobile Learning System Based on Digital Campus," Journal of Southwest China Normal University (Natural Science Edition), vol. 39, no. 5, pp. 59-63, 2014.

[5] J. Lu, "Design and Realization of College English Mobile Learning Platform Based on Mobile Phone," Master's degree of Anhui Normal University, 2012.

[6] Q. Z. Yu, L. Bai, J. J. Guo, "Study on Application and System Construction of Campus: based Mobile Learning Platform," Science and Technology Management Research, vol. 34, no. 13, pp. 126-130, 2014.

[7] A. M. Wu, "Wutong_login column: HLS protocol implementation," http://blog.csdn.net/wutong_login/article/details/9673255, 2016-2-14. 